\title{
Isoliquiritigenin inhibits the proliferation of human renal carcinoma Caki cells through the ROS-mediated regulation of the Jak2/STAT3 pathway
}

\author{
DO-HEE KIM ${ }^{1 *}$, JI EUN PARK ${ }^{2 *}$, IN GYEONG CHAE ${ }^{2}$, GEUMI PARK $^{2}$, \\ SOOYEUN LEE ${ }^{2}$ and KYUNG-SOO $\mathrm{CHUN}^{2}$ \\ ${ }^{1}$ College of Pharmacy, Seoul National University, Seoul 151-742; \\ ${ }^{2}$ College of Pharmacy, Keimyung University, Daegu 704-701, Republic of Korea
}

Received November 29, 2016; Accepted April 24, 2017

DOI: $10.3892 /$ or.2017.5677

\begin{abstract}
Isoliquiritigenin (ISL) is a flavonoid with chalcone structure that has been noted in licorice and shallot, which are generally used in traditional Chinese medicine. ISL has demonstrated various pharmacological effects including antioxidant, anti-inflammatory and antitumor activity. However, the molecular mechanisms underlying the anticancer effects of ISL remain poorly understood. The present study revealed that ISL significantly decreased viability and induced apoptosis in human renal carcinoma Caki cells. The ISL-induced apoptosis was associated with the cleavage of caspase-9, -7 and -3 , and that of PARP. Moreover, ISL increased the expression of pro-apoptotic protein Bax and diminished the expression of anti-apoptotic protein $\mathrm{Bcl}-2$, and $\mathrm{Bcl}-\mathrm{xl}$, thereby increasing cytochrome $c$ release. Treatment of cells with ISL also induced the expression of p53 through downregulation of murine double minute 2 (Mdm2). Furthermore, ISL generated reactive oxygen species (ROS), and pretreatment with ROS scavenger $\mathrm{N}$-acetyl cysteine (NAC) and NADPH oxidase inhibitor diphenyleneiodonium abrogated the ISL-induced apoptosis. One of the key oncogenic signaling pathways is mediated through signal transducer and activator of transcription 3 (STAT3), which promotes abnormal cell proliferation. Incubation of cells with ISL markedly diminished phosphorylation and DNA binding activity of STAT3, and reduced expression of STAT3 responsive gene products, such as cyclin D1 and D2. ISL also attenuated constitutive phosphorylation of upstream
\end{abstract}

Correspondence to: Professor Kyung-Soo Chun or Professor Sooyeun Lee, College of Pharmacy, Keimyung University, 1095 Dalgubeoldaero, Dalseo-Gu, Daegu 704-701, Republic of Korea E-mail: chunks@kmu.ac.kr

E-mail: sylee21@kmu.ac.kr

${ }^{*}$ Contributed equally

Key words: isoliquiritigenin, apoptosis, reactive oxygen species, signal transducer and activator of transcription 3, renal cancer kinase, Janus-activated kinase 2 (Jak2). Pretreatment with NAC abrogated the inhibitory effect of ISL on activation of STAT3 and blocked the cleavage of caspase-9, -7 and -3, and that of PARP in Caki cells. Taken together, the present study provides the first report that ISL induces apoptosis in Caki cells via generation of ROS, which causes induction of p53 and inhibition of the STAT3 signaling pathway.

\section{Introduction}

Renal cell carcinoma ( $\mathrm{RCC}$ ) is the most common type of malignant neoplasm arising from the kidney (1). RCC has the highest mortality rate of all genitourinary cancer and its incidence has steadily risen (2). Since RCC is resistant to conventional chemotherapy, it commonly recurs after treatment; thus, patients with metastatic RCC have poor prognosis (3). Typically, the drugs used to treat metastatic RCC are targeted to vascular endothelial growth factor (VEGF) receptors and mammalian target of rapamycin (mTOR) signaling. However, while most patients initially respond to these drugs, its resistance results in subsequent progression of RCC. Thus, to address the unmet need to identify additional targets for RCC, newly developed therapeutic agents are required for effective treatment.

Signal transducer and activator of transcription 3 (STAT3) is a transcriptional factor that mediates the signaling pathway of various cytokines and growth factors (4). Constitutive activation of STAT3 has been observed in human cancer including breast, ovarian and prostate cancer (5-7). It has been recently reported that a high frequency of STAT3 activation is observed in RCC, particularly in metastatic disease (8). STAT3 protein has been shown to play an important role in supporting cell survival and proliferation (9). In cancer cells, STAT3 becomes constitutively active through the phosphorylation of its serine 727 (S727) and tyrosine 705 (Y705) residues by upstream Janus-activated kinases (Jaks) or the Src family kinases (9). Phosphorylated STAT3 then undergoes dimerization and is translocated to the nucleus where it binds to specific DNA response elements in the promoters of target genes (10). STAT3 transactivates the genes encoding various cell survival proteins, such as cyclins, survivin, Bcl-2 and 
Bcl-xl (11). This evidence supports the rationale for targeting STAT3 for suppression of tumor growth in several cancers including RCC (12).

Apoptosis is triggered by extrinsic and/or intrinsic pathways and is regulated by various ligands (13). The extrinsic pathway originates from interaction between death receptors and its ligands such as Fas and $\mathrm{TNF} \alpha$. The intrinsic pathway is closely related to the permeabilization of the mitochondrial membrane, commanded by the Bcl-2 superfamily (14). Reactive oxygen species (ROS) are mainly generated by the mitochondria in most mammalian cells. ROS can cause cellular apoptosis via both the extrinsic cell death receptor and the intrinsic mitochondrial cell death pathways (15). It has been reported that elevated ROS formation and the subsequent apoptosis induction is a novel approach to treat cancer (16).

Since evasion of apoptosis is one of the hallmarks of cancer, it has long been attempted to develop anticancer drugs that can selectively induce apoptosis in cancer cells (17). Various phytochemicals such as polyphenols modulate differentiation of cells and induce apoptosis in cancer cells (18). Isoliquiritigenin [2',4',4-trihydroxychalcone (ISL)] (Fig. 1A) is a flavonoid with chalcone structure that has been noted in licorice and shallot, which are generally used in traditional Chinese medicine (19). ISL shows pharmacological effects including antioxidant, anti-inflammatory and antitumor activity (20-22). In addition, ISL has been proved to induce apoptosis and inhibit cell proliferation in breast, liver, colon and cervical cancer cells (23-27). Moreover, ISL was found to inhibit tumor growth and angiogenesis in lung and breast cancer mouse models $(22,28)$. Previously, Yamazaki et al reported that ISL suppressed the viability of murine RCC with concomitant inhibition of nitric oxide production by lipopolysaccharide-stimulated macrophages, resulting in decreased pulmonary metastasis (29). However, thus far, there have been no studies regarding the effects of ISL in human RCC. Therefore, we aimed to investigate molecular mechanisms underlying the anticancer effects of ISL on human renal carcinoma Caki cells. The present study revealed that ISL induced antiproliferative and apoptotic effects in Caki cells through mitochondrial-dependent caspase activation and interference with the STAT3 signaling pathway via ROS generation.

\section{Materials and methods}

Materials. ISL, $N$-acetyl cysteine (NAC), diphenyleneiodonium (DPI) and $\beta$-actin antibody were purchased from Sigma-Aldrich Co. (St. Louis, MO, USA). Antibodies against cleaved caspase-9, -7 -3 and PARP as well as Bcl-2, Bcl-xl, Bax, STAT3, p-STAT3 (Y705), p-STAT3 (S727), Jak2, p-Jak2, cyclin D1 and cyclin D2 were obtained from Cell Signaling Technology, Inc. (Beverly, MA, USA). Primary antibodies against each of p53 and murine double minute-2 (Mdm2), and horseradish peroxidase-conjugated secondary antibodies were purchased from Santa Cruz Biotechnology (Santa Cruz, CA, USA). 2'-7'-Dichlorofluorescein diacetate (DCF-DA) was obtained from Invitrogen (Carlsbad, CA, USA). Hank's balanced salt solution (HBSS) was purchased from Mediatech (Herndon, VA, USA).
Cell culture and treatment. Caki cells were provided by Dr. T.K. Kwon (Keimyung University, Daegu, Korea) and maintained in Dulbecco's modified Eagle's medium (DMEM) supplemented with $10 \%$ fetal bovine serum and antibiotics (100 U/ml penicillin $\mathrm{G}$ and $100 \mu \mathrm{g} / \mathrm{ml}$ streptomycin) at $37^{\circ} \mathrm{C}$ in a humidified incubator containing 5\% $\mathrm{CO}_{2}$ and $95 \%$ air. The cells were plated at an appropriate density according to each experimental scale.

Cell viability assay. Cell viability was measured by the MTT assay. Cells $\left(2 \times 10^{3}\right)$ were incubated in triplicate in a 96-well plate in the presence of ISL in a final volume of $100 \mu 1$ for different time intervals at $37^{\circ} \mathrm{C}$. Thereafter, $10 \mu \mathrm{l}$ of MTT solution $(5 \mathrm{mg} / \mathrm{ml})$ was added to each well and incubated for $4 \mathrm{~h}$. Medium was removed, formazan was dissolved in dimethyl sulfoxide (DMSO) and absorbance at $570 \mathrm{~nm}$ was measured using a microplate reader (Tecan Trading AG, Männedorf, Switzerland). Cell viability was expressed as the relative percentage of the control.

Annexin V staining. Annexin V staining was performed using fluorescein isothiocyanate (FITC)-Annexin V staining kit (BD Biosciences, San Jose, CA, USA) following the manufacturer's instructions. Briefly, ISL-treated cells were washed with HBSS solution and resuspended in binding buffer containing Annexin V and propidium iodide (PI). Fluorescence intensity was measured using flow cytometry (BD Biosciences, La Jolla, CA, USA).

Western blot analysis. Cells were lysed with RIPA buffer, and collected protein samples were quantified using a bicinchoninic acid protein assay kit (Pierce Biotechnology, Rockford, IL, USA). The protein samples were electrophoresed using 8-15\% sodium dodecyl sulfate-polyacrylamide gel and immunoblot analysis was carried out according to the protocol previously described (30). Immunoblot membranes were incubated with SuperSignal Pico Chemiluminescent or Dura Luminol substrates (Thermo Fisher Scientific, Inc., Waltham, MA, USA) according to the manufacturer's instruction and visualized with ImageQuant LAS 4000 (Fujifilm Life Science, Tokyo, Japan).

Measurement of ROS accumulation. Cells were treated with ISL in the presence or absence of NAC and DPI for $24 \mathrm{~h}$ and then loaded with $25 \mu \mathrm{M}$ of DCF-DA. After incubation for $30 \mathrm{~min}$ at $37^{\circ} \mathrm{C}$, the cells were washed twice with HBSS solution, suspended in complete media and examined under a fluorescence microscope to detect the intracellular accumulation of ROS. Total cellular ROS were quantified using a flow cytometry (BD Biosciences).

Electrophoretic mobility gel shift assay (EMSA). The nuclear extract was prepared from cells incubated with or without ISL. The STAT3 oligonucleotide probe 5'-AGC TTC ATT TCC CGT AAA TCC CTA-3' (Biomedic, Korea) was labeled with $\left[\gamma_{-}{ }^{32} \mathrm{P}\right]-\mathrm{ATP}$ using T4 polynucleotide kinase. The EMSA was performed according to a previously described protocol (31).

Statistical analysis. When necessary, data are expressed as mean \pm SD of the results of at least three independent 

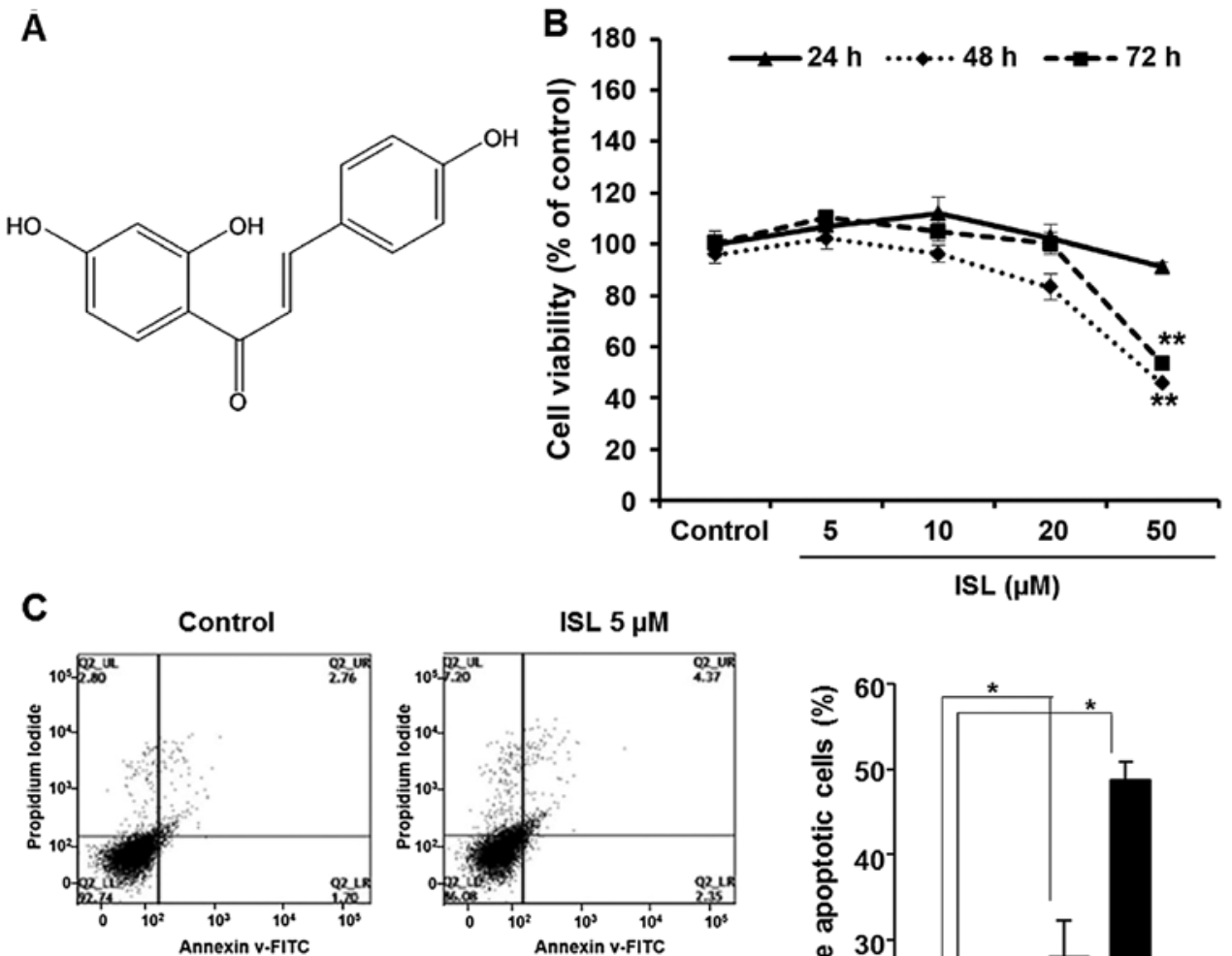

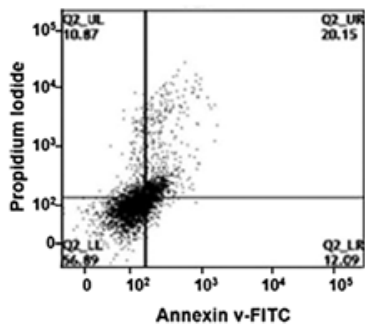

ISL $20 \mu \mathrm{M}$

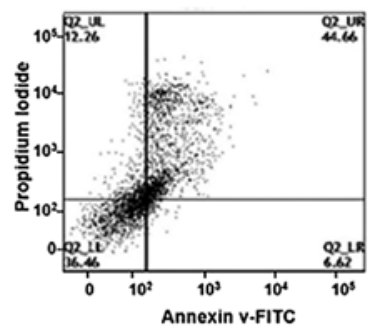

ISL $50 \mu \mathrm{M}$
ISL $(\mu \mathrm{M})$

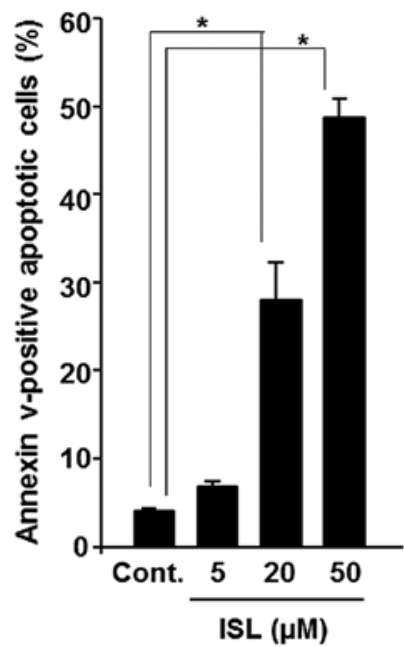

Figure 1. Cytotoxic effect of ISL in Caki cells. (A) Chemical structure of ISL. (B) Caki cells were treated with the indicated concentrations of ISL for 24, 48 or $72 \mathrm{~h}$. Cell viability was determined by the MTT assay. (C) Cells were treated with the indicated concentrations of ISL for $48 \mathrm{~h}$ and the apoptotic index (\%) was analyzed by flow cytometry. Cells were stained with FITC-conjugated anti-Annexin V antibody and PI. The graph shows statistical analysis of apoptosis. Data are representative of three independent experiments; ${ }^{*} \mathrm{p}<0.05,{ }^{* *} \mathrm{p}<0.001$, compared to the control.

experiments, and statistical analysis for single comparison was performed using the Student's t-test and a p-value $<0.05$ was considered to indicate a statistically significant result.

\section{Results}

ISL induces apoptosis in Caki cells. We initially examined the effect of ISL on the viability of Caki cells. Incubation of cells with ISL $(5,10,20$ or $50 \mu \mathrm{M})$ significantly reduced the cell viability (Fig. 1B). In addition, to elucidate whether ISL-mediated cytotoxicity resulted from the induction of apoptosis, FACS analysis was performed. Caki cells treated with ISL $(5,20$ or $50 \mu \mathrm{M})$ for $48 \mathrm{~h}$ were analyzed by flow cytometry using double staining with Annexin V and PI to quantify the population of cells undergoing apoptosis. As shown in Fig. 1C, incubation of Caki cells with ISL for $48 \mathrm{~h}$ resulted in apoptotic cell death. Quantification of apoptotic cells and statistical analysis of ISL-induced apoptosis are presented in Fig. 1C (right panel).
ISL induces apoptosis through the mitochondrial pathway in Caki cells. Mitochondria are a central regulator of the intrinsic pathway in apoptosis triggered by various stimuli (32). The loss of mitochondrial membrane potential leads to increased mitochondrial membrane permeability, and then released cytochrome $c$, which induces activation of the caspase cascade (32). The activation of caspases is a critical event in the proteolytic cascade elicited by apoptotic stimuli. We investigated the involvement of caspase activation in the ISL-induced apoptosis of Caki cells. Treatment of Caki cells with ISL induced the cleavage of caspase- $9,-7$ and -3 , and PARP (Fig. 2A). Incubation of cells with ISL increased the expression of p53 and diminished the expression of its cytosolic repressor protein $\mathrm{Mdm} 2$ in a concentration-dependent manner (Fig. 2B). To elucidate whether the apoptosis may be mediated by mitochondrial alterations, we evaluated the expression of anti-apoptotic or pro-apoptotic proteins in the ISL-treated Caki cells. Anti-apoptotic proteins such as Bcl-2 and $\mathrm{Bcl}-\mathrm{xl}$ reside in the outer mitochondrial membrane and 
A

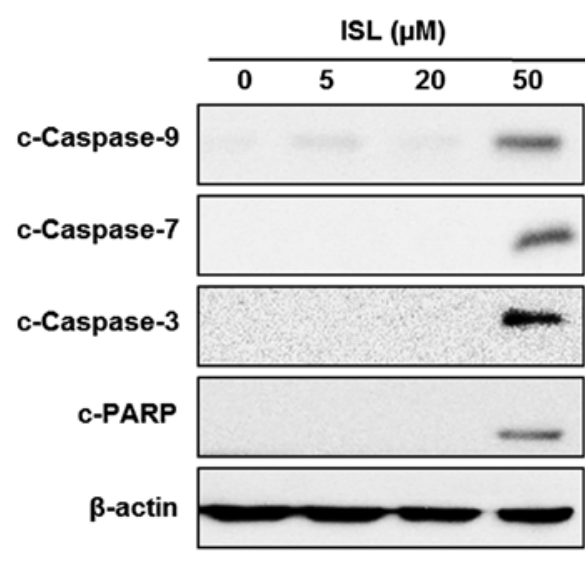

B
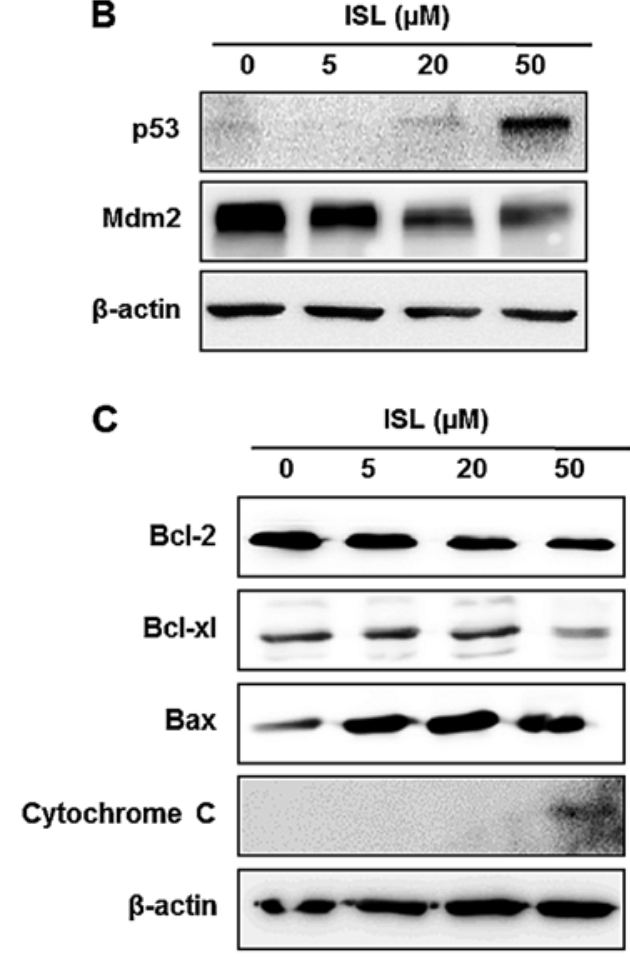

Figure 2. Effect of ISL on cellular markers of apoptosis in Caki cells. Caki cells were treated with the indicated concentrations of ISL for 48 h. (A) Immunoblot analysis for detecting the levels of cleaved caspase-9, -7 and -3 , and cleaved PARP. (B) Western blot analysis of p53 and Mdm2 expression after incubation of Caki cells with ISL. (C) The expression level of Bcl-2, Bcl-xl, Bax and cytochrome $c$. Data are representative of three different experiments. $\beta$-Actin was used as a loading control.

block the release of cytochrome $c$, whereas pro-apoptotic protein Bax stimulates its release. Since Bcl-2 family proteins regulate the mitochondrial membrane integrity, the effect of ISL on the expression of Bcl-2 family proteins was then examined. As shown in Fig. 2C, incubation with ISL reduced the expression of Bcl-2 and Bcl-xl, while ISL increased expression of Bax in Caki cells. In addition, the level of cytochrome $c$ expression was increased by treatment of ISL in the Caki cells with a concomitant decrease in Bcl-2 expression (Fig. 2C).

ISL induces generation of ROS in Caki cells. Since it has been postulated that ROS production contributes to apoptosis triggering in various cancer cell lines (33), the effect of ISL on ROS generation was examined. Treatment of cells with ISL (5, 20 or $50 \mu \mathrm{M}$ ) for $24 \mathrm{~h}$ led to ROS generation as revealed by FACS analysis after DCF-DA staining (Fig. 3A). As a positive control, Caki cells were incubated with hydrogen peroxide $(500 \mu \mathrm{M})$ under the same experimental condition. To verify that the increase in ROS level was involved in the ISL-induced apoptosis, we utilized the ROS scavenger NAC and specific NADPH oxidase inhibitor DPI. It has been known that NADPH oxidases catalyze the production of superoxide, a type of ROS. Treatment of Caki cells with NAC and DPI abrogated the ISL-induced ROS generation (Fig. 3B and C), following cell death (Fig. 3D and E). Quantification of apoptotic cell death under each experimental condition as well as statistical analysis are presented in the right panel of Fig. 3D and E.

ISL-induced apoptosis in Caki cells is mediated through inactivation of STAT3 signaling. STAT3 protein is constitu- tively activated in various human cancer cells and aberrant STAT3 signaling is implicated as playing a pivotal role in the initiation and progression of cancer (34). It has been known that STAT3 transcriptional activity is stimulated by its simultaneous tyrosine and serine phosphorylation via activation of Jak kinases (35). Incubation with ISL inhibited the STAT3 DNA binding activity following inhibition of constitutive phosphorylation of STAT3 at both Y705 and S727 residues (Fig. 4A and B). To elucidate the mechanism of ISL-induced inactivation of STAT3, we examined the effect of ISL on the phosphorylation of upstream kinase, Jak2, which is known to phosphorylate STAT3. As shown in Fig. 4A, ISL attenuated the constitutive phosphorylation of Jak2 in Caki cells. In addition, ISL attenuated the expression of STAT3 target gene products such as cyclin D1 and cyclin D2 (Fig. 4C).

Role of ROS in ISL-induced inhibition of STAT3 signaling and induction of apoptosis in Caki cells. We next examined the role of ISL-induced ROS generation in the blocking of STAT3 signaling and induction of apoptosis in Caki cells. Incubation of Caki cells with ISL in the absence or presence of NAC revealed that inhibition of ROS generation abrogated the inhibitory effect of ISL on the phosphorylation of Jak2 following STAT3 activation (Fig. 5A) as well as its DNA binding activity (Fig. 5B). Moreover, pretreatment of NAC averted the ISL-induced cleavage of caspase-9 -7 and -3 and PARP (Fig. 5C). These findings suggest that the ISL-induced ROS generation plays a pivotal role in apoptosis and inactivation of STAT3 signaling in the ISL-treated Caki cells. 
A

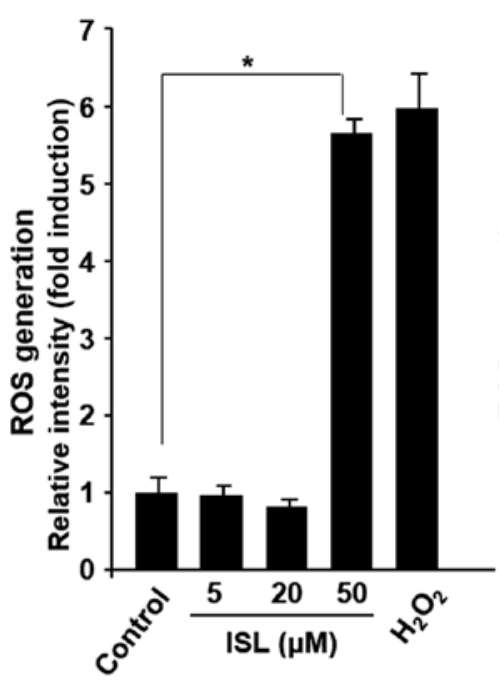

B

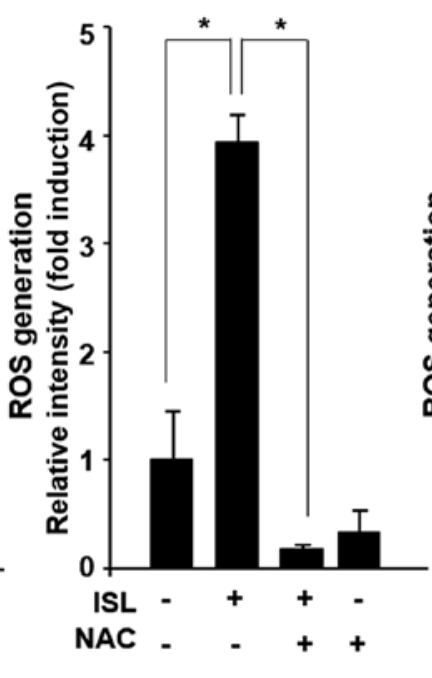

C

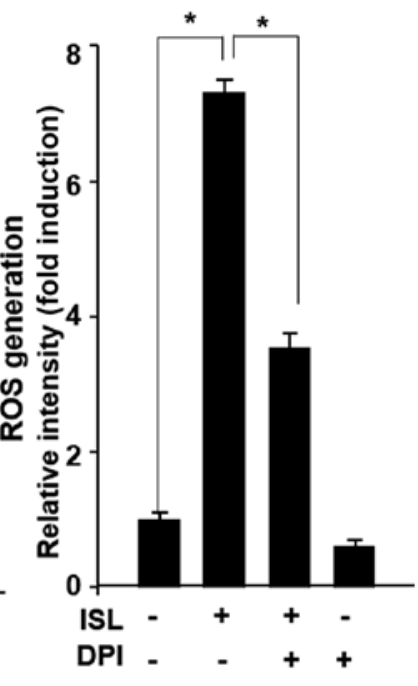

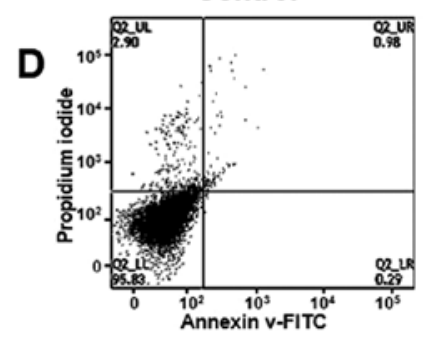

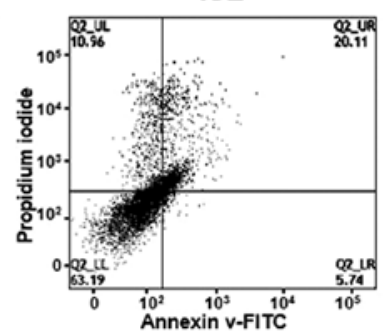

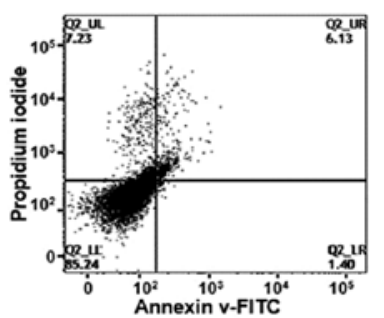

ISL + NAC

E
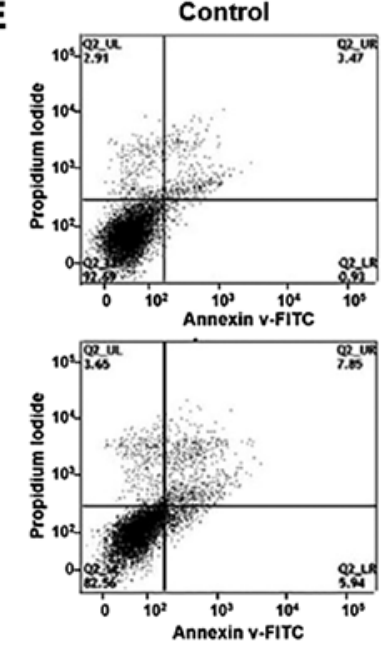

ISL + DPI

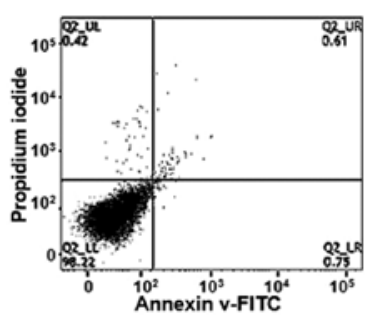

NAC
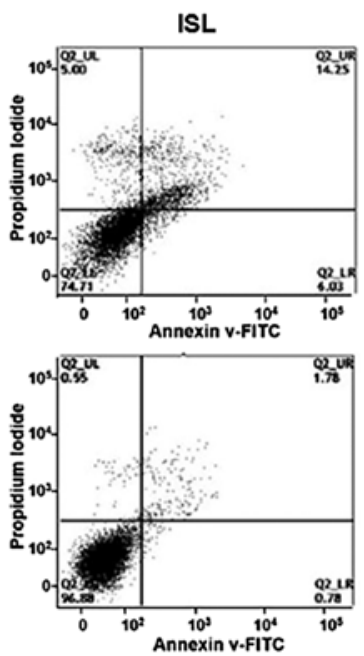

DPI
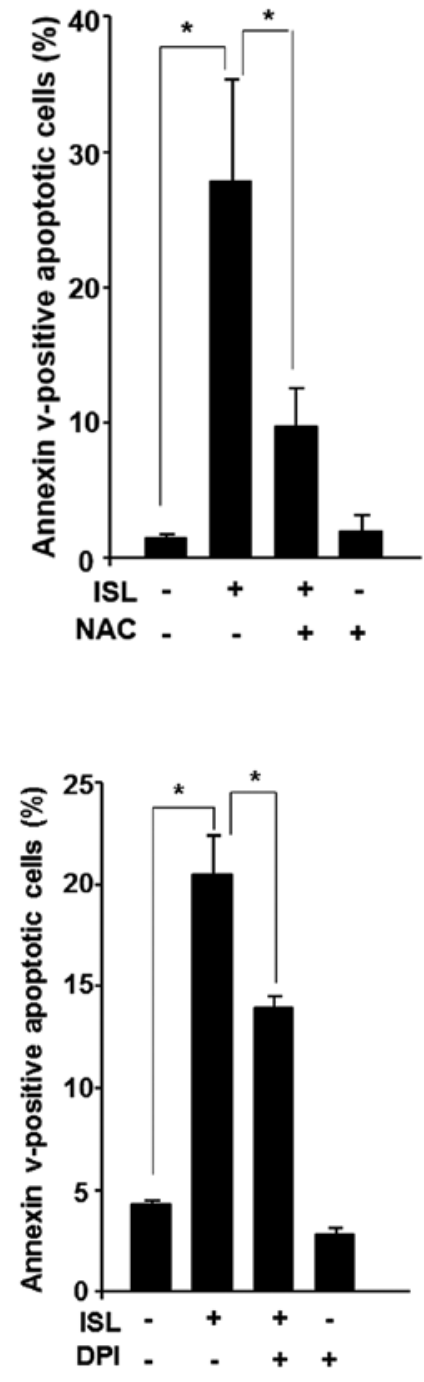

Figure 3. ISL induces ROS generation in Caki cells. (A) Cells were treated with ISL (5, 20 or $50 \mu \mathrm{M})$ for $24 \mathrm{~h}$, and then examined for the intracellular accumulation of ROS. DCF-DA fluorescence staining was measured by flow cytometry. Hydrogen peroxide $\left(\mathrm{H}_{2} \mathrm{O}_{2}\right)(500 \mu \mathrm{M})$ was used as a positive control. (B and C) Effect of antioxidants on ISL-generated ROS in Caki cells. Cells were treated with NAC (5 mM) and DPI (50 nM) for $1 \mathrm{~h}$ before treatment with ISL $(50 \mu \mathrm{M})$ for $24 \mathrm{~h}$. ROS levels were measured using flow cytometry. This graph shows quantification and statistical analysis of ROS generation by ISL treatment in the presence or absence of (B) NAC and (C) DPI. The experiment was carried out in triplicate and the data are presented as mean \pm SD. (D and E) Roles of ROS in ISL-induced apoptosis in Caki cells. Cells were treated with NAC (5 mM) and DPI (50 nM) for $1 \mathrm{~h}$ before treatment with ISL (50 $\mu \mathrm{M})$ for $48 \mathrm{~h}$. The apoptotic index $(\%)$ was determined using flow cytometry. This graph shows statistical analysis of apoptosis by ISL treatment in the presence or absence of (D) NAC and (E) DPI. Data are representative of three independent experiments; ${ }^{* *} \mathrm{p}<0.001$, compared to control. 
A

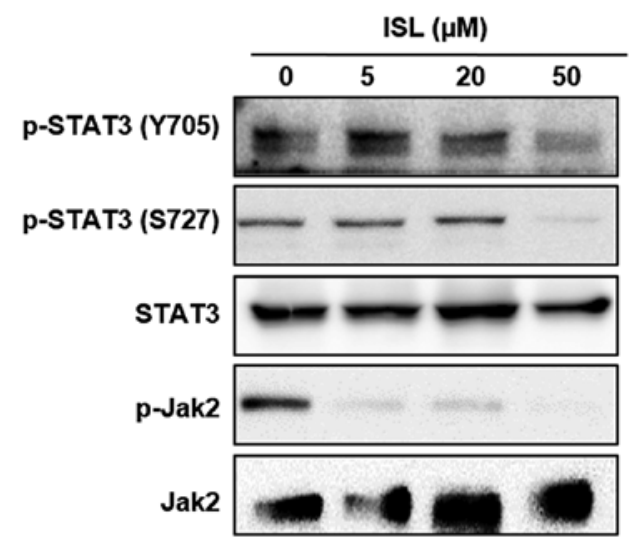

B

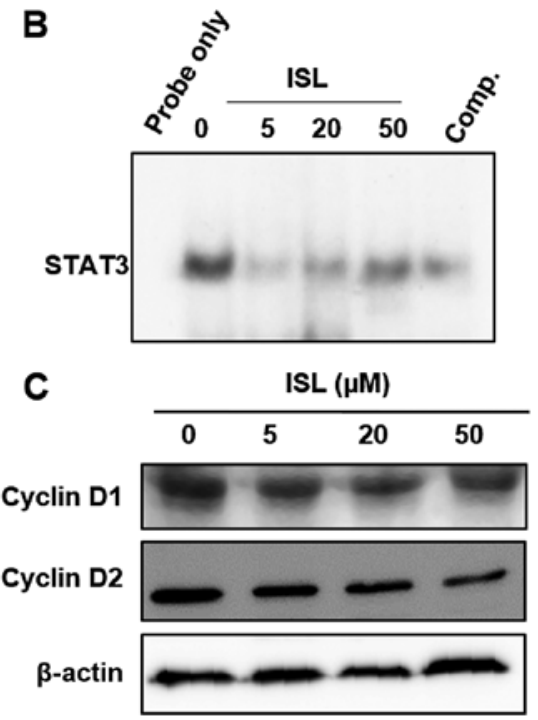

Figure 4. ISL inhibits constitutive activation of STAT3 and expression of its gene products. (A) Cells were treated with the indicated concentrations of ISL and the level of p-Jak2, p-STAT3 (Y705) and p-STAT3 (S727) was detected using western blot assay. (B) Nuclear extract prepared from cells treated with ISL was assessed for the DNA binding of STAT3. For competition, molar excess of unlabeled cold STAT3 oligo was used. (C) Cells were treated with the indicated concentrations of ISL for $48 \mathrm{~h}$. Immunoblot analysis was performed to assess the expression of cyclin D1 and cyclin D2.
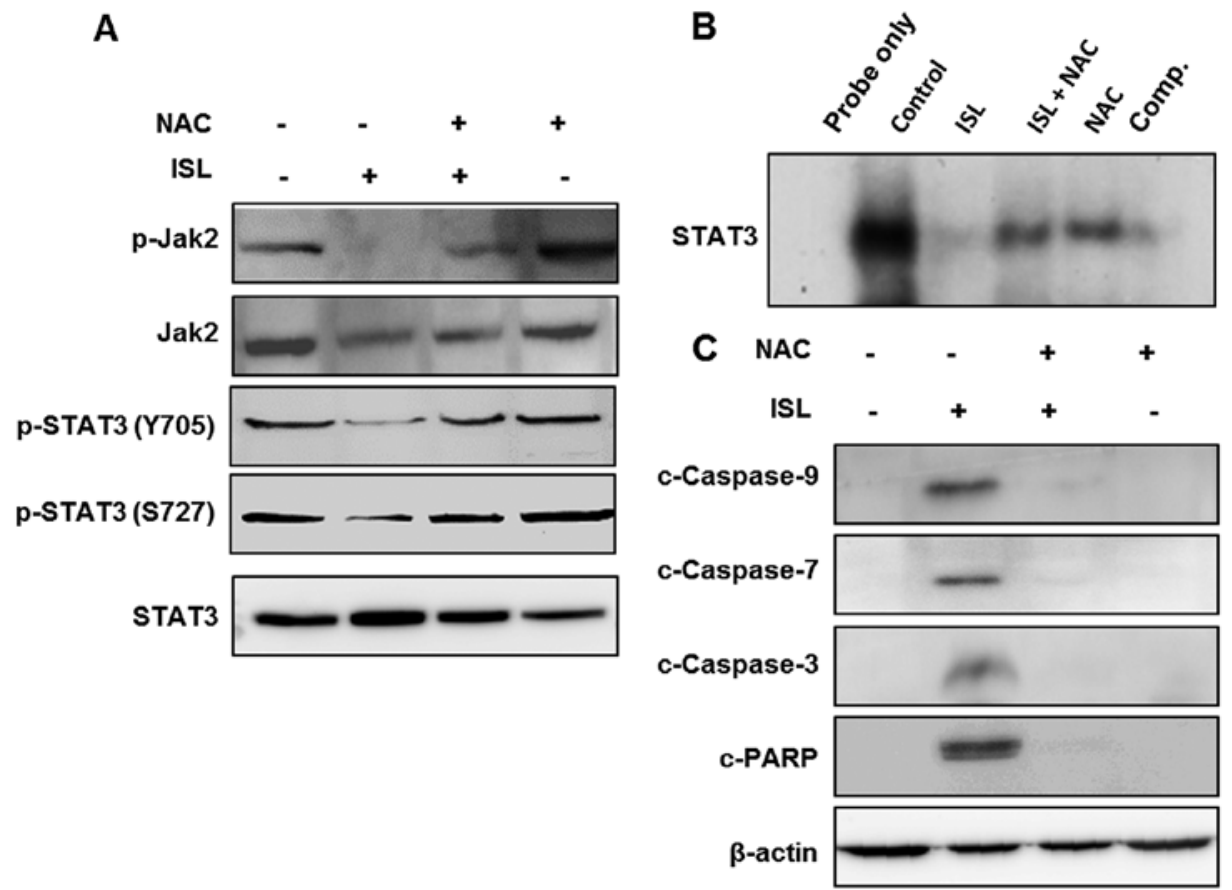

Figure 5. Roles of ROS in ISL-induced inactivation of STAT3 signaling and apoptosis in Caki cells. Cells were treated with ISL (50 $\mu \mathrm{M})$ for $48 \mathrm{~h}$ after pre-incubation with or without NAC (5 mM) for $1 \mathrm{~h}$. (A) Levels of p-STAT3 (Y705), p-STAT3 (S727) and p-Jak2 were detected by western blot analysis. (B) Nuclear extract prepared from cells treated with NAC $(5 \mathrm{mM}) 1 \mathrm{~h}$ before treatment with ISL $(50 \mu \mathrm{M})$ for $48 \mathrm{~h}$ was assessed for the DNA binding of STAT3. (C) Cleaved forms of PARP, caspase- $9,-7$ and -3 protein were detected using immunoblot analysis.

\section{Discussion}

Approaches to RCC therapy have many limitations and novel strategies are urgently required. It has been reported that dysregulated cell growth is a hallmark of cancer development, and naturally occurring substances derived from plant-based diet have been suggested for used in the therapy of various types of cancers (36). In addition, Jak/STAT3 signaling controls the ability of pre-neoplastic lesions to transform into a malignant tumor, which suggests that intervention of the Jak/STAT3 pathway provides the opportunities for new approaches in cancer therapy (37). Cumulative evidence supports activation of STAT3 as an oncogenic pathway in many cancers including RCC (5-8). To address the unmet need to identify therapeutic 


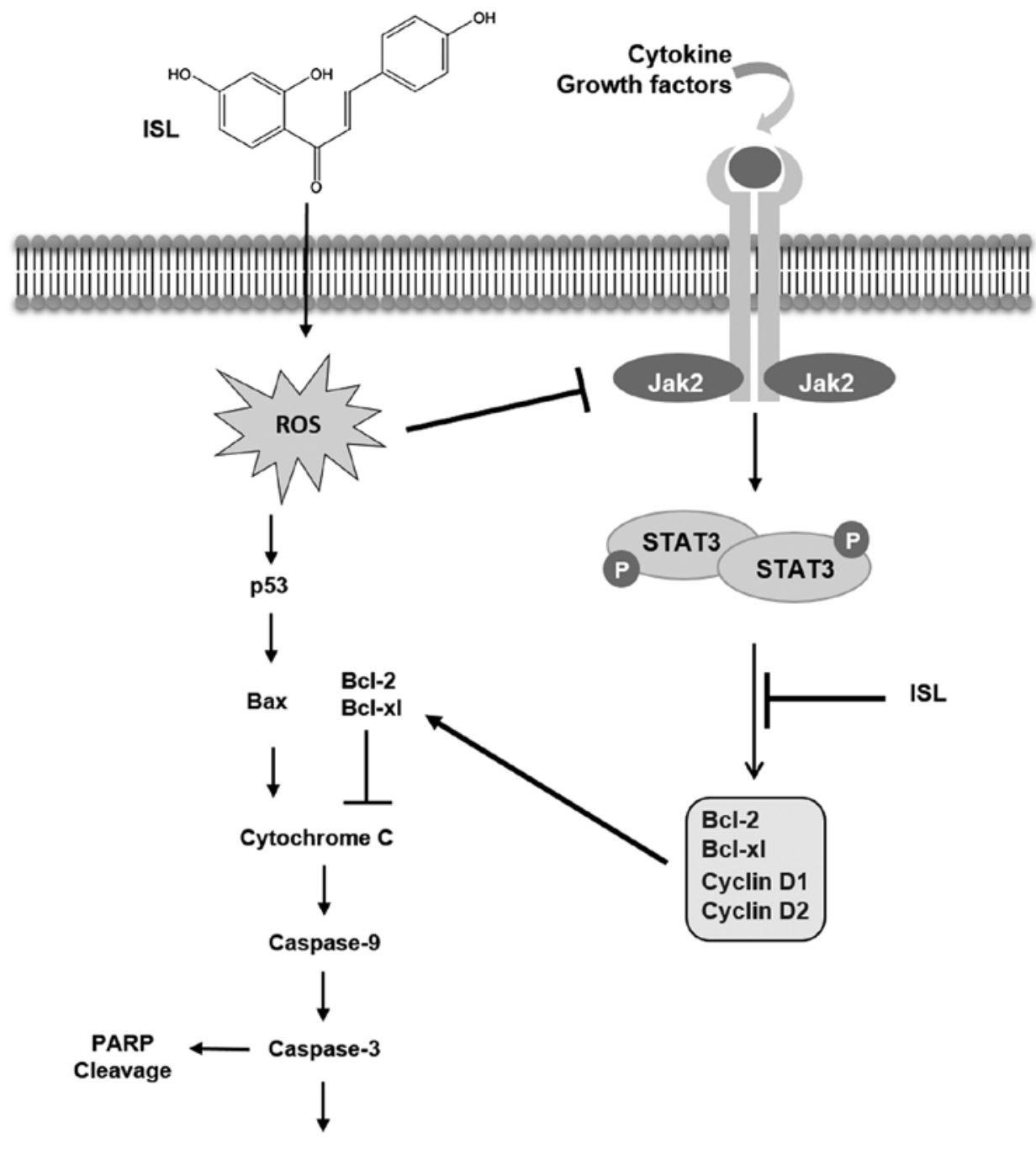

Apoptosis

Figure 6. A schematic diagram showing the molecular mechanisms of apoptosis induction by ISL in Caki cells.

targets for RCC, researchers have recently identified the Jak/ STAT3 pathway. Our group and other investigators have suggested that kinase inhibitors of this pathway and phytochemicals suppressing STAT3 cause marked tumor growth inhibition in RCC in vitro and in vivo models $(38,39)$.

Chalcone possesses a basic structure of two benzene rings connected by an unsaturated carbon chain and are the intermediate precursors for all flavonoid compounds. ISL is a chalcone found in licorice root and other plants, and shows various pharmacological properties including antitumor, antioxidant, and anti-allergic activities in vitro (40-43). ISL also contains an $\alpha, \beta$-unsaturated carbonyl group as a Michael acceptor site, suggesting that ISL-induced ROS is mediated through the depletion of intracellular GSH. The electrophilic center such as the $\alpha, \beta$-unsaturated carbonyl group is prone to undergo Michael addition reactions with nucleophiles, such as the free sulfhydryl group of cysteine residues located in reduced glutathione or many cellular proteins (44). In the present study, we provide the novel finding that ISL attenuated the constitutive phosphorylation, nuclear localization and DNA binding activity of STAT3 in Caki cells. Thus, we propose that ISL may directly bind to GSH and/or cysteine residues of STAT3. Phosphorylation of STAT3 initiates their nuclear translocation, followed by activated STAT3 dimers, and binds to specific promoter regions in DNA (45). In addition, it was reported that stattic, a small molecular inhibitor of STAT3, prevented STAT3 dimerization and phosphorylation, and this was accompanied by alkylation of four cysteine residues (Cys251, 259, 367 and 426) in unphosphorylated STAT3 (46). Moreover, it has been shown that specific cysteine residues of STAT3 undergo covalent modifications and/or oxidation, and then changes in the cellular redox status affect the transcriptional activity of STAT3 (47). It is likely that ISL-induced ROS may cause oxidative modification of cysteine residues of Jak2 or STAT3 proteins, thereby blunting the survival of Caki cells. Our findings suggest that pretreatment with NAC abrogated the inhibitory effects of ISL on the activation of Jak2/STAT3 signaling implying that ISL can induce oxidative modification of these kinases. Similar to this finding, natural products such as withaferin A and carnosol were found to induce apoptosis through inhibition of Jak2/STAT3, accompanied by generation of $\operatorname{ROS}(48,49)$.

The role of ROS in the apoptosis of cancer cells is quite controversial. Although some investigators suspect that ROS 
play a role in pro-tumorigenic signaling, other investigators have reported that ROS induce apoptosis (50). It seems likely that many antitumor agents cause oxidative stress such as ROS, which also contributes to their anticancer effects (51). Li et al revealed that ROS induce apoptosis through suppression of Bcl-2 expression and an increase in the Bax protein level in squamous cell carcinoma cells (52). The present study, also demonstrated that upregulation of the $\mathrm{Bax} / \mathrm{Bcl}-2$ ratio may contribute to the apoptosis in ISL-stimulated Caki cells. In addition, NAC treatment rescued cells from ISL-induced apoptosis by blocking the cleavage of caspases and PARP. Schumacker explained that cancer cells may be more vulnerable to oxidative stress, since they function with a higher level of ROS-mediated growth signaling than normal cells (53). Therefore, ISL has important value in the treatment of RCC, based on induction of oxidative stress-mediated apoptotic signals.

Recent studies have confirmed that the Jak2/STAT3 pathways are implied in the regulation of apoptosis and survival of tumor cells. STAT3 can also enhance its DNA binding activity by activating growth factors such as IL-6 and IL-17 to regulate the transcription of the corresponding target genes. It continuously transduces the survival signal to tumor cells to play a role in the promotion of tumor cell survival and inhibition of apoptosis (54). Therefore, in the present study, the effects of ISL on activation of STAT3 were estimated, and inhibition of the Jak2/STAT3 pathway resulted in apoptosis in ISL-treated Caki cells. In addition, ISL downregulated the expression of STAT3-related anti-apoptotic and survival genes such as Bcl-2, Bcl-xl, cyclin D1 and D2 in the Caki cells. Intracellular kinases, such as Jak2, have been shown to phosphorylate STAT3. The inhibitory effect of ISL on the phosphorylation of Jak 2 through ROS generation interfered with STAT3 signaling pathway in the Caki cells. STAT3 becomes constitutively active through the phosphorylation by Src kinase as well as Jak. Moreover, a synergistic effect on tumor inhibition by co-treatment of Src and STAT3 inhibitors in RCC was noted (38). In accordance with these studies, we found that incubation of Caki cells with ISL inhibited Src activation and pretreatment with NAC abrogated the inhibitory effect of ISL on the phosphorylation of Src activation (data not shown). These data imply the role of Src in STAT3 activation in ISL-treated Caki cells. In summary, the present study demonstrated for the first time that ISL induces apoptosis in Caki cells through generation of ROS, which causes induction of p53 and inhibition of the STAT3 signaling pathway (Fig. 6).

\section{Acknowledgements}

The present study was supported by the Basic Science Research Program through the National Research Foundation of Korea (NRF) funded by the Ministry of Education (NRF-2016R1A6A1A03011325), and by the Bio and Medical Technology Development Program of NRF funded by the Korean Government, MSIP (2015M3A9B6073827).

\section{References}

1. Grimm MO, Wolff I, Zastrow S, Fröhner M and Wirth M: Advances in renal cell carcinoma treatment. Ther Adv Urol 2: $11-17,2010$.
2. De P, Otterstatter MC, Semenciw R, Ellison LF, Marrett LD and Dryer D: Trends in incidence, mortality, and survival for kidney cancer in Canada, 1986-2007. Cancer Causes Control 25: 1271-1281, 2014.

3. Motzer RJ, Michaelson MD, Redman BG, Hudes GR, Wilding G, Figlin RA, Ginsberg MS, Kim ST, Baum CM, DePrimo SE, et al: Activity of SU11248, a multitargeted inhibitor of vascular endothelial growth factor receptor and platelet-derived growth factor receptor, in patients with metastatic renal cell carcinoma. J Clin Oncol 24: 16-24, 2006.

4. Darnell JE Jr: STATs and gene regulation. Science 277: 1630-1635, 1997.

5. Dhir R, Ni Z, Lou W, DeMiguel F, Grandis JR and Gao AC: Stat3 activation in prostatic carcinomas. Prostate 51: 241-246, 2002.

6. Huang M, Page C, Reynolds RK and Lin J: Constitutive activation of stat 3 oncogene product in human ovarian carcinoma cells. Gynecol Oncol 79: 67-73, 2000.

7. Proietti CJ,Rosemblit C,Beguelin W, Rivas MA,Díaz Flaqué MC Charreau EH, Schillaci R and Elizalde PV: Activation of Stat3 by heregulin/ErbB-2 through the co-option of progesterone receptor signaling drives breast cancer growth. Mol Cell Biol 29: $1249-1265,2009$.

8. Guo C, Yang G, Khun K, Kong X, Levy D, Lee P and Melamed J: Activation of Stat3 in renal tumors. Am J Transl Res 1: 283-290, 2009.

9. Yu H, Lee H, Herrmann A, Buettner R and Jove R: Revisiting STAT3 signalling in cancer: New and unexpected biological functions. Nat Rev Cancer 14: 736-746, 2014

10. Bowman T, Garcia R, Turkson J and Jove R: STATs in oncogenesis. Oncogene 19: 2474-2488, 2000.

11. Johnston PA and Grandis JR: STAT3 signaling: Anticancer strategies and challenges. Mol Interv 11: 18-26, 2011.

12. Carpenter RL and Lo HW: STAT3 target genes relevant to human cancers. Cancers 6: 897-925, 2014.

13. Portt L, Norman G, Clapp C, Greenwood M and Greenwood MT: Anti-apoptosis and cell survival: A review. Biochim Biophys Acta 1813: 238-259, 2011.

14. Riedl SJ and Salvesen GS: The apoptosome: Signalling platform of cell death. Nat Rev Mol Cell Biol 8: 405-413, 2007.

15. Sinha K, Das J, Pal PB and Sil PC: Oxidative stress: The mitochondria-dependent and mitochondria-independent pathways of apoptosis. Arch Toxicol 87: 1157-1180, 2013.

16. Cerna D, Li H, Flaherty S, Takebe N, Coleman CN and Yoo SS: Inhibition of nicotinamide phosphoribosyltransferase (NAMPT) activity by small molecule GMX1778 regulates reactive oxygen species (ROS)-mediated cytotoxicity in a p53- and nicotinic acid phosphoribosyltransferase1 (NAPRT1)-dependent manner. J Biol Chem 287: 22408-22417, 2012.

17. Hanahan D and Weinberg RA: Hallmarks of cancer: The next generation. Cell 144: 646-674, 2011.

18. Kampa M, Nifli AP, Notas G and Castanas E: Polyphenols and cancer cell growth. Rev Physiol Biochem Pharmacol 159: 79-113, 2007.

19. Aida K, Tawata M, Shindo H, Onaya T, Sasaki H, Yamaguchi T, Chin M and Mitsuhashi H: Isoliquiritigenin: A new aldose reductase inhibitor from Glycyrrhizae Radix. Planta Med 56: 254-258, 1990

20. Choi YH, Bae JK, Chae HS, Choi YO, Nhoek P, Choi JS and Chin YW: Isoliquiritigenin ameliorates dextran sulfate sodium-induced colitis through the inhibition of MAPK pathway. Int Immunopharmacol 31: 223-232, 2016.

21. Gaur R, Yadav KS, Verma RK, Yadav NP and Bhakuni RS: In vivo anti-diabetic activity of derivatives of isoliquiritigenin and liquiritigenin. Phytomedicine 21: 415-422, 2014.

22. Jung SK, Lee MH, Lim DY, Kim JE, Singh P, Lee SY, Jeong CH, Lim TG, Chen H, Chi YI, et al: Isoliquiritigenin induces apoptosis and inhibits xenograft tumor growth of human lung cancer cells by targeting both wild type and L858R/T790M mutant EGFR. J Biol Chem 289: 35839-35848, 2014.

23. Auyeung KK and Ko JK: Novel herbal flavonoids promote apoptosis but differentially induce cell cycle arrest in human colon cancer cell. Invest New Drugs 28: 1-13, 2010.

24. Jung JI, Chung E, Seon MR, Shin HK, Kim EJ, Lim SS, Chung WY, Park KK and Park JH: Isoliquiritigenin (ISL) inhibits ErbB3 signaling in prostate cancer cells. Biofactors 28: 159-168, 2006.

25. Lai PH, Li KT, Hsu SS, Hsiao CC, Yip CW, Ding S, Yeh LR and Pan HB: Pyogenic brain abscess: Findings from in vivo 1.5-T and 11.7-T in vitro proton MR spectroscopy. AJNR Am J Neuroradiol 26: 279-288, 2005. 
26. Li Y, Zhao H, Wang Y, Zheng H, Yu W, Chai H, Zhang J, Falck JR, Guo AM, Yue J, et al: Isoliquiritigenin induces growth inhibition and apoptosis through downregulating arachidonic acid metabolic network and the deactivation of PI3K/Akt in human breast cancer. Toxicol Appl Pharmacol 272: 37-48, 2013.

27. Park I, Park KK, Park JH and Chung WY: Isoliquiritigenin induces G2 and M phase arrest by inducing DNA damage and by inhibiting the metaphase/anaphase transition. Cancer Lett 277: 174-181, 2009.

28. Wang Z, Wang N, Han S, Wang D, Mo S, Yu L, Huang H, Tsui K, Shen J and Chen J: Dietary compound isoliquiritigenin inhibits breast cancer neoangiogenesis via VEGF/VEGFR-2 signaling pathway. PLoS One 8: e68566, 2013.

29. Yamazaki S, Morita T, Endo H, Hamamoto T, Baba M, Joichi Y, Kaneko S, Okada Y, Okuyama T, Nishino H, et al: Isoliquiritigenin suppresses pulmonary metastasis of mouse renal cell carcinoma. Cancer Lett 183: 23-30, 2002.

30. Chae IG, Kim DH, Kundu J, Jeong CH, Kundu JK and Chun KS Generation of ROS by CAY 10598 leads to inactivation of STAT3 signaling and induction of apoptosis in human colon cancer HCT116 cells. Free Radic Res 48: 1311-1321, 2014.

31. Kim DH, Park KW, Chae IG, Kundu J, Kim EH, Kundu JK and Chun KS: Carnosic acid inhibits STAT3 signaling and induces apoptosis through generation of ROS in human colon cancer HCT116 cells. Mol Carcinog 55: 1096-1110, 2016.

32. Indran IR, Tufo G, Pervaiz S and Brenner C: Recent advances in apoptosis, mitochondria and drug resistance in cancer cells. Biochim Biophys Acta 1807: 735-745, 2011.

33. Simon HU, Haj-Yehia A and Levi-Schaffer F: Role of reactive oxygen species (ROS) in apoptosis induction. Apoptosis 5 415-418, 2000

34. Bromberg J: Stat proteins and oncogenesis. J Clin Invest 109: $1139-1142,2002$

35. Aznar S, Valerón PF, del Rincon SV, Pérez LF, Perona R and Lacal JC: Simultaneous tyrosine and serine phosphorylation of STAT3 transcription factor is involved in Rho A GTPase oncogenic transformation. Mol Biol Cell 12: 3282-3294, 2001.

36. Bishayee A and Sethi G: Bioactive natural products in cancer prevention and therapy: Progress and promise. Semin Cancer Biol 40-41: 1-3, 2016

37. Arumuggam N, Bhowmick NA and Rupasinghe HP: A Review: Phytochemicals Targeting JAK/STAT Signaling and IDO Expression in Cancer. Phytother Res 29: 805-817, 2015.

38. Lue HW, Cole B, Rao SA, Podolak J, Van Gaest A, King C, Eide CA, Wilmot B, Xue C, Spellman PT, et al: Src and STAT3 inhibitors synergize to promote tumor inhibition in renal cell carcinoma. Oncotarget 6: 44675-44687, 2015.

39. Um HJ, Min KJ, Kim DE and Kwon TK: Withaferin A inhibits JAK/STAT3 signaling and induces apoptosis of human renal carcinoma Caki cells. Biochem Biophys Res Commun 427: 24-29, 2012.

40. Chin YW, Jung HA, Liu Y, Su BN, Castoro JA, Keller WJ, Pereira MA and Kinghorn AD: Anti-oxidant constituents of the roots and stolons of licorice (Glycyrrhiza glabra). J Agric Food Chem 55: 4691-4697, 2007.
41. Kakegawa $\mathrm{H}$, Matsumoto $\mathrm{H}$ and Satoh $\mathrm{T}$ : Inhibitory effects of some natural products on the activation of hyaluronidase and their anti-allergic actions. Chem Pharm Bull 40: 1439-1442, 1992.

42. Kang SW, Choi JS, Choi YJ, Bae JY, Li J, Kim DS, Kim JL, Shin SY, Lee YJ and Kwun IS: Licorice isoliquiritigenin dampens angiogenic activity via inhibition of MAPK-responsive signaling pathways leading to induction of matrix metalloproteinases. J Nutr Biochem 21: 55-65, 2010.

43. Kape R, Parniske M, Brandt S and Werner D: Isoliquiritigenin, a strong nod gene- and glyceollin resistance-inducing flavonoid from soybean root exudate. Appl Environ Microbiol 58: 1705-1710, 1992.

44. Deponte M: Glutathione catalysis and the reaction mechanisms of glutathione-dependent enzymes. Biochim Biophys Acta 1830 3217-3266, 2013.

45. Bromberg J and Darnell JE Jr: The role of STATs in transcriptional control and their impact on cellular function. Oncogene 19: 2468-2473, 2000

46. Heidelberger S, Zinzalla G, Antonow D, Essex S, Basu BP, Palmer J, Husby J, Jackson PJ, Rahman KM, Wilderspin AF, et al: Investigation of the protein alkylation sites of the STAT3:STAT3 inhibitor Stattic by mass spectrometry. Bioorg Med Chem Lett 23: 4719-4722, 2013

47. Li L, Cheung SH, Evans EL and Shaw PE: Modulation of gene expression and tumor cell growth by redox modification of STAT3. Cancer Res 70: 8222-8232, 2010.

48. Park KW, Kundu J, Chae IG, Kim DH, Yu MH, Kundu JK and Chun KS: Carnosol induces apoptosis through generation of ROS and inactivation of STAT3 signaling in human colon cancer HCT116 cells. Int J Oncol 44: 1309-1315, 2014.

49. Sinha P and Ostrand-Rosenberg S: Myeloid-derived suppressor cell function is reduced by Withaferin A, a potent and abundant component of Withania somnifera root extract. Cancer Immunol Immunother 62: 1663-1673, 2013.

50. Sullivan LB and Chandel NS: Mitochondrial reactive oxygen species and cancer. Cancer Metab 2: 17, 2014.

51. Pan MH, Sin YH, Lai CS, Wang YJ, Lin JK, Wang $M$ and Ho CT: Induction of apoptosis by 1-(2-hydroxy-5-methylphenyl)3-phenyl-1,3-propanedione through reactive oxygen species production, GADD153 expression, and caspases activation in human epidermoid carcinoma cells. J Agric Food Chem 53: 9039-9049, 2005.

52. Li D, Ueta E, Kimura T, Yamamoto T and Osaki T: Reactive oxygen species (ROS) control the expression of Bcl-2 family proteins by regulating their phosphorylation and ubiquitination. Cancer Sci 95: 644-650, 2004.

53. Schumacker PT: Reactive oxygen species in cancer cells: Live by the sword, die by the sword. Cancer Cell 10: 175-176, 2006.

54. Jiang YQ, Zhou ZX and Ji YL: Suppression of EGFR-STAT3 signaling inhibits tumorigenesis in a lung cancer cell line. Int J Clin Exp Med 7: 2096-2099, 2014. 